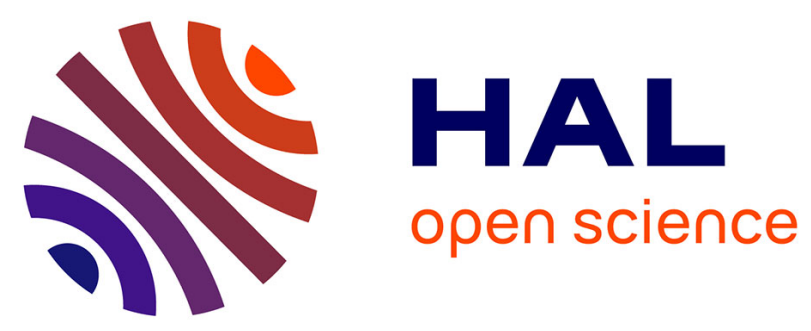

\title{
Microwave and dc response of an Abrikosov vortex lattice in ferromagnetic superconductors
}

\author{
A. A. Bespalov, A. S. Mel'Nikov, Alexandre I. Buzdin
}

\section{To cite this version:}

A. A. Bespalov, A. S. Mel'Nikov, Alexandre I. Buzdin. Microwave and dc response of an Abrikosov vortex lattice in ferromagnetic superconductors. Physica C: Superconductivity and its Applications, 2014, 503, pp.98-100. 10.1016/j.physc.2014.04.046 . hal-01061709

\section{HAL Id: hal-01061709 \\ https://hal.science/hal-01061709}

Submitted on 26 Feb 2015

HAL is a multi-disciplinary open access archive for the deposit and dissemination of scientific research documents, whether they are published or not. The documents may come from teaching and research institutions in France or abroad, or from public or private research centers.
L'archive ouverte pluridisciplinaire HAL, est destinée au dépôt et à la diffusion de documents scientifiques de niveau recherche, publiés ou non, émanant des établissements d'enseignement et de recherche français ou étrangers, des laboratoires publics ou privés. 


\title{
Microwave and dc response of an Abrikosov vortex lattice in ferromagnetic superconductors
}

\author{
A.A. Bespalov ${ }^{\mathrm{a}, \mathrm{b}, *}$, A.S. Mel'nikov ${ }^{\mathrm{a}, \mathrm{c}}$, A.I. Buzdin ${ }^{\mathrm{b}}$ \\ a Institute for Physics of Microstructures, Russian Academy of Sciences, GSP-105, 603950 Nizhny Novgorod, Russia \\ ${ }^{\mathrm{b}}$ Université Bordeaux, CNRS, LOMA, UMR 5798, F-33400 Talence, France \\ c Nizhny Novgorod State University, 22 Gagarin av., 603950 Nizhny Novgorod, Russia
}

Keywords:

Ferromagnetic superconductors

Surface impedance

Vortex dynamics

\begin{abstract}
A B S T R A C T
In magnetic superconductors the magnetic flux dynamics is influenced by the interaction of vortices with the magnetization. This interaction leads to the appearance of an additional damping force acting on the vortices. By solving the London and Landau-Lifshitz-Gilbert equations we analyze the ac and dc responses of a ferromagnetic superconductor in the mixed state. If the vortices are driven by an ac force, their viscosity is enhanced due to the generation of magnons. This viscosity enhancement affects the surface impedance of the sample. In the case of a dc driving current vortices start to radiate magnons when their velocity exceeds a threshold value. As a result, either a step-like feature or a series of peaks appear on the $I-V$ curve.
\end{abstract}

Within the last 13 years the coexistence of ferromagnetism and superconductivity has been discovered in several compounds [1]: $\mathrm{UGe}_{2}$ [2] (with a superconducting transition temperature $\left.T_{c}=0.7 \mathrm{~K}\right)$, URhGe [3] $\left(T_{c}=0.25 \mathrm{~K}\right)$, UCoGe [4-6] $\left(T_{c}=0.8 \mathrm{~K}\right)$, and doped $\mathrm{EuFe}_{2} \mathrm{As}_{2}$ [7] $\left(T_{c}=25 \mathrm{~K}\right)$. These compounds offer a unique possibility to study the interplay between superconductivity and ferromagnetism in the bulk. A manifestation of this interplay is the modification of the spin wave (magnon) spectrum by the superconductivity. This effect has been investigated theoretically by Buzdin in the case of superconducting antiferromagnet [8]. For a ferromagnetic superconductor in the Meissner state, different types of spin waves have been previously analyzed, including bulk magnons [9,10], domain wall waves [11] and surface waves [12].

Experimental evidence of the incomplete Meissner effect in the ferromagnetic superconductors $[3,4,6]$ suggests that they exhibit a spontaneous vortex state even in the absence of an external magnetic field. So far only two papers have addressed the magnon spectrum in the mixed state of a ferromagnetic superconductor $[13,14]$. Ng and Varma [13] studied long-wavelength magnons: then, the Abrikosov vortex lattice can be treated within the continuous medium approximations. In the limit of short wavelengths [14], the magnon spectrum acquires a Bloch-like band structure due to the Bragg scattering on the vortex lattice.

* Corresponding author at: Institute for Physics of Microstructures, Russian Academy of Sciences, GSP-105, 603950 Nizhny Novgorod, Russia. Tel.: +7 9026886209 .

E-mail address: bespalovaa@gmail.com (A.A. Bespalov).
The reverse influence of magnetism on vortex dynamics has been considered by Bulaevskii et al. in a series of papers [15-19]. It has been demonstrated theoretically that magnons can be excited by moving vortices [15-17] (then, the magnon spectrum can be extracted from the current-voltage characteristics at different values of the applied magnetic field), and in systems with slow relaxation of the magnetization a polaronic mechanism of selfinduced vortex pinning exists $[18,19]$. Finally, it has been shown, using numeric simulations, that moving vortices should create domain walls in magnetic materials with a sufficiently large relaxation time of the magnetic moments [20].

The preceding papers [15-19] mainly concentrated on flux dynamics in superconducting antiferromagnets. In the present paper we analyze the influence of the vortex-magnetic moment interaction on the microwave and dc responses of a ferromagnetic superconductor in the mixed state (the Meissner state has been considered in $[9,10])$.

A possible geometry for the experimental study of the microwave response of a ferromagnetic superconductor is shown in Fig. 1. Here, the surface impedance of the sample is measured. We assume that the vortices are perpendicular to the sample surface. It will be important for us that the magnetic field $\mathbf{h}_{e}$ of the probing electromagnetic wave be parallel to the magnetization easy axis (directed along the vector $\mathbf{M}_{0}$ ), so that magnon modes propagating along the $z$-axis are not excited (see Refs. $[9,10,14]$ ). Then, in a wide range of parameters the following simple expression for the surface impedance is valid [21]: 


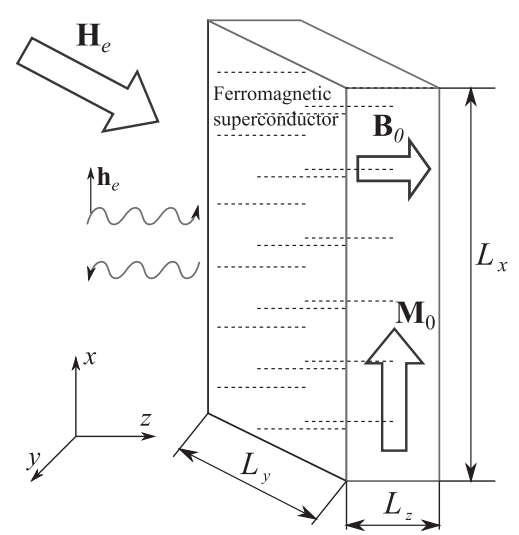

Fig. 1. The geometry for the measurement of the surface impedance of a ferromagnetic superconductor. $\mathbf{H}_{e}$ is a static external magnetic field. The vector $\mathbf{M}_{0}$ is directed along the magnetization easy axis. The dashed lines denote vortices.

$Z(\omega)=\left(\frac{-i \omega \mu B_{0} \Phi_{0}}{4 \pi c^{2}\left(\eta+\eta_{M}\right)}\right)^{1 / 2}$

where $\omega$ is the frequency of the probing field, $\mu$ is the static differential magnetic permeability, $B_{0}$ is the internal magnetic field, $\Phi_{0}$ is the flux quantum, $\eta$ is the vortex viscosity due to order parameter relaxation processes and normal current flowing through the vortex core [22], and $\eta_{M}$ is a complex contribution to the viscosity originating from the interaction of vortices with the magnetization. In the present work the quantity $\eta_{M}$ has been calculated by solving the phenomenological Landau-Lifshitz-Gilbert and London equations.

In the London approximation the free energy of the ferromagnetic superconductor can be taken in the form [23]

$F=\int\left[\frac{1}{8 \pi \lambda^{2}}\left(\mathbf{A}+\frac{\Phi_{0}}{2 \pi} \nabla \theta_{S}\right)^{2}+\frac{(\operatorname{rot} \mathbf{A}-4 \pi \mathbf{M})^{2}}{8 \pi}+\frac{\alpha}{2}\left(\frac{\partial \mathbf{M}}{\partial x_{i}} \frac{\partial \mathbf{M}}{\partial x_{i}}\right)+\frac{K \mathbf{M}_{\perp}^{2}}{2}\right] d^{3} \mathbf{r}$.

Here $\lambda$ is the London penetration depth, $\mathbf{A}$ is the vector potential, $\theta_{S}$ is the superconducting order parameter phase, $\mathbf{M}$ is the magnetization, and $\alpha$ is a constant characterizing the exchange interaction. The U-based ferromagnetic superconductors have a strong easyaxis magnetocrystalline anisotropy, which is accounted for by the term $K \mathbf{M}_{\perp}^{2} / 2$, where $K \gg 1$ is an anisotropy constant, $\mathbf{M}_{\perp}=\mathbf{M}-(\mathbf{e} \cdot \mathbf{M}) \mathbf{e}$, and $\mathbf{e}$ is a unit vector along the anisotropy axis. Within the London theory, the vector potential should be determined from the equation $\delta F / \delta \mathbf{A}=0$.

For simplicity, we assume that the Abrikosov vortices are straight and aligned along the $z$-axis, which makes an angle $\theta$ with the magnetization easy axis. The vortices may form a regular or disordered lattice. When the vortices are set in motion by an external current, the time-dependent magnetic field induces magnetization fluctuations, which can be described using the Landau-Lifshitz-Gilbert equation [24]

$\frac{\partial \mathbf{M}}{\partial t}=\gamma\left(\mathbf{M} \times \frac{\delta F}{\delta \mathbf{M}}\right)+\frac{v}{M^{2}}\left(\mathbf{M} \times \frac{\partial \mathbf{M}}{\partial t}\right)$,

where $\gamma$ is the gyromagnetic ratio and $v$ is a dissipation constant.

In our model the magnetic moment induced force acting on a unit length of a vortex is

$\mathbf{f}_{M}=-\frac{1}{N_{v}} \int M_{z} \nabla h_{z} d^{2} \boldsymbol{\rho}$,

where $N_{v}$ is the number of vortices, $h_{z}$ is the $z$-component of the vortex field, and integration is over the $x y$-plane. Note that $\mathbf{f}_{M}$ is the force averaged over all vortices. If the vortices are driven by a harmonic transport current, in the linear regime $\mathbf{f}_{M}$ can be written as
$\mathbf{f}_{M}=-\eta_{M} \dot{\mathbf{R}} e^{-i \omega t}-\eta_{M}^{*} \dot{\mathbf{R}}^{*} e^{i \omega t}$,

where $\dot{\mathbf{R}}$ is the amplitude of the vortex velocity oscillations. Eqs. (5) gives the definition of the viscosity coefficient $\eta_{M}$ entering Eq. (1).

For sufficiently clean samples, when the vortices form an almost ideal lattice, we obtained

$$
\begin{aligned}
\eta_{M}= & -\frac{i \gamma M \Phi_{0} B_{0}}{2 \omega} \sin ^{2} \theta \sum_{\mathbf{G}} \frac{G^{2}}{\left(1+\lambda^{2} G^{2}\right)^{2}} \\
& \times\left[\frac{\omega(G)}{\omega^{2}(G)-\omega^{2}-2 i \frac{v}{M} \omega \omega(G)}-\omega^{-1}(G)\right] .
\end{aligned}
$$

where summation is over all vectors $\mathbf{G}$ of the reciprocal lattice, $\omega(G)=\omega_{F}\left(1+L^{2} G^{2}\right)$ is the magnon spectrum, $\omega_{F}=\gamma M K$ is the ferromagnetic resonance frequency, and $L=\sqrt{\alpha / K}$ is of the order of the Bloch domain wall width (it is just a length scale, we consider a monodomain system). The $\eta_{M}$ vs. $B_{0}$ dependencies for a dissipation rate $v / M=0.02$ and different frequencies $\omega>\omega_{F}$ are shown in Fig. 2. The Lorentzian-like $\left(\mathfrak{R}\left(\eta_{M}\right)\right)$ and $\mathrm{N}$-shaped $\left(\mathfrak{J}\left(\eta_{M}\right)\right)$ features correspond to resonances at $\omega(G)=\omega$. According to Eq. (6), the peak values of $\mathfrak{R}\left(\eta_{M}\right)$ are proportional to $\omega^{-2}$. Thus, to make the resonances more pronounced compounds with low values of the ferromagnetic resonance frequency are preferable.

For a sample with randomly pinned vortices we obtained the expression

$$
\begin{aligned}
& \eta_{M}=\frac{\gamma M \Phi_{0}^{2} \sin ^{2} \theta}{8 \pi \omega \omega_{F} \lambda^{4}}\left\{\frac{\pi \omega_{F}}{2\left(\omega-\omega_{F}\right)} \Theta\left(\omega-\omega_{F}\right)\right. \\
& \left.-i\left[\frac{2 \omega^{2}}{\omega_{F}^{2}-\omega^{2}} \ln \frac{\lambda}{L}+\frac{\omega_{F}}{2\left(\omega+\omega_{F}\right)} \ln \frac{\omega+\omega_{F}}{\omega_{F}}+\frac{\omega_{F}}{2\left(\omega_{F}-\omega\right)} \ln \left|\frac{\omega_{F}-\omega}{\omega_{F}}\right|\right]\right\} .
\end{aligned}
$$

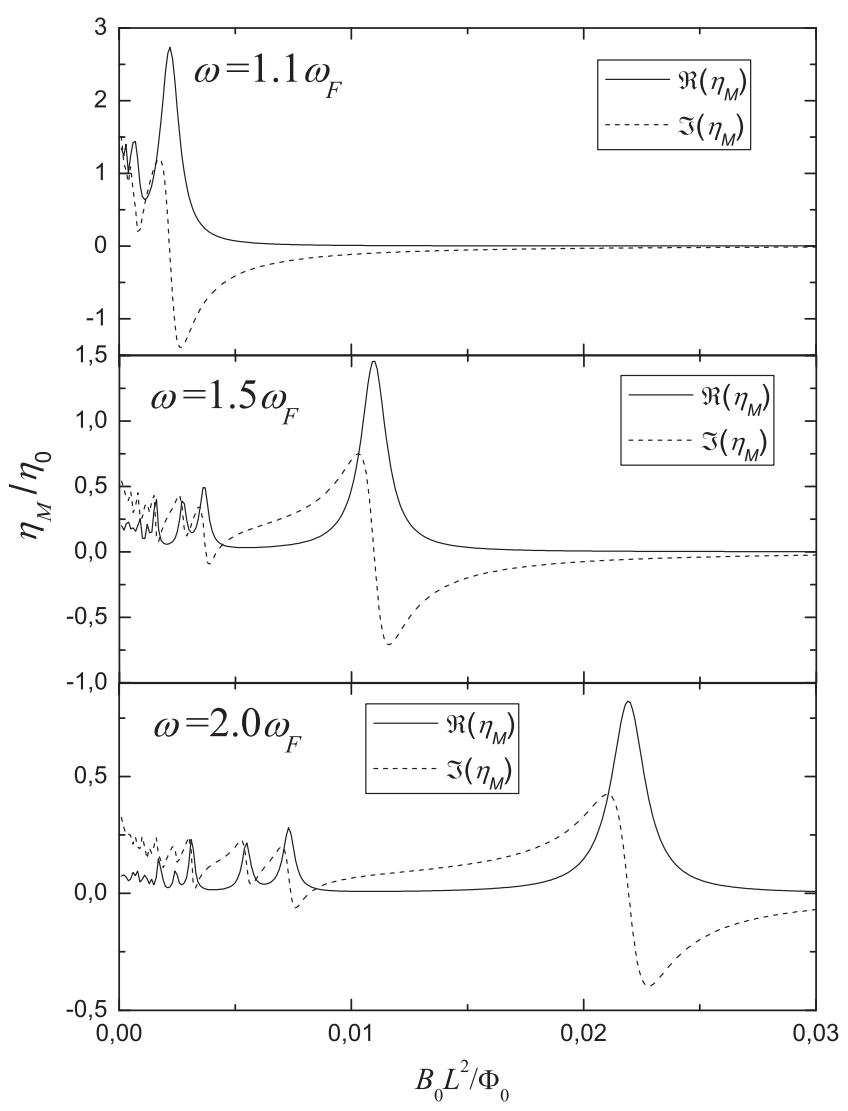

Fig. 2. The $\eta_{M}$ vs. magnetic field dependencies for frequencies above the ferromagnetic resonance frequency (see Eq. (6)). $\eta_{0}=\gamma M \Phi_{0}^{2} \sin ^{2} \theta /\left(2 \lambda^{4} \omega_{F}^{2}\right)$. The vortices form an ideal triangular lattice. 


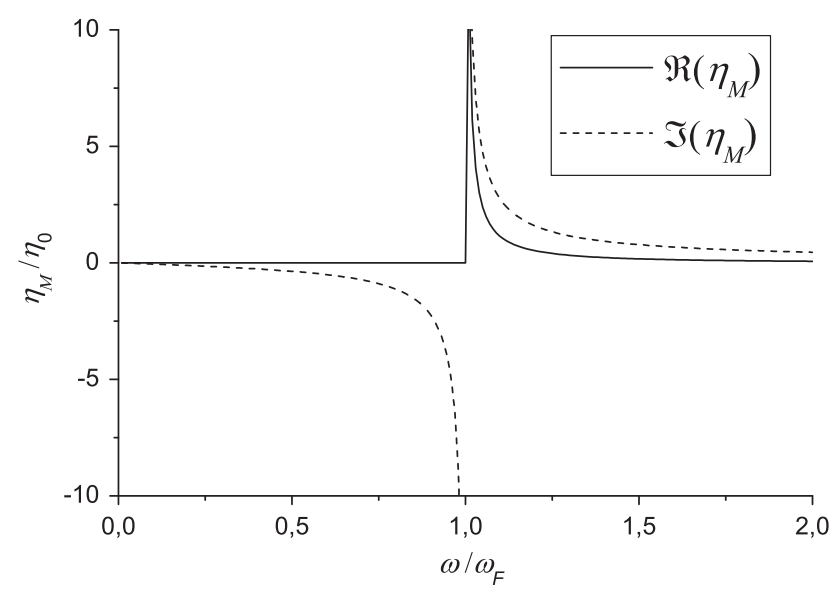

Fig. 3. The frequency dependence of the magnetic viscosity, $\eta_{M}$, for a disordered vortex array - see Eq. (7). The value $\ln (\lambda / L)=4.3$ of $\mathrm{UGe}_{2}$ has been used. $\eta_{0}=\gamma M \Phi_{0}^{2} \sin ^{2} \theta /\left(2 \lambda^{4} \omega_{F}^{2}\right)$.

For this case the $\eta_{M}$ vs. $\omega$ dependence is depicted in Fig. 3.

If the frequency of the driving current is low, or its amplitude is large, the vortex response is essentially non-linear. We determined this response in the strongly non-linear regime, when the sample is biased by a dc current. In the case of an ideal vortex lattice, the magnetic moment induced damping force is

$$
\begin{aligned}
\mathbf{f}_{M}= & -\gamma \nu B_{0} \Phi_{0} \sin ^{2} \theta \sum_{\mathbf{G}} \frac{\mathbf{G}\left(\mathbf{G} \mathbf{V}_{L}\right)}{\left(1+\lambda^{2} G^{2}\right)^{2}} \\
& \times \frac{\left(\mathbf{G V}_{L}\right)^{2}+\omega^{2}(G)}{\left[\omega^{2}(G)-\left(\mathbf{G V}_{L}\right)^{2}\right]^{2}+4 \frac{v^{2}}{M^{2}}\left(\mathbf{G V}_{L}\right)^{2} \omega^{2}(G)},
\end{aligned}
$$

where $\mathbf{V}_{L}$ is the flux velocity. Eq. (8) has two important consequences. First, the force has local maxima when for some vector $\mathbf{G}=\mathbf{G}_{0}$ the Cherenkov resonance condition $\omega\left(G_{0}\right) \approx \mathbf{V}_{L} \mathbf{G}_{\mathbf{0}}$ is satisfied. The resonances should lead to the appearance of peaks on the current-voltage characteristics at electric fields $\mathbf{E}$ satisfying $\omega\left(G_{0}\right)-c / B_{0}\left(\mathbf{z}_{0} \times \mathbf{G}_{0}\right) \mathbf{E} \approx 0$. Measurements of the peak voltages at different applied magnetic fields allow to determine the magnon spectrum of the ferromagnetic superconductor. Second, it can be seen from Eq. (8) that $\mathbf{f}_{M}$ is generally not parallel to the flux velocity $\mathbf{V}_{L}$. It follows from this that the vortex-magnetic moment interaction gives an anisotropic contribution to the flux-flow conductivity.

Finally, we considered the case of a disordered vortex lattice, which may appear in relatively weak magnetic field, $B_{0} \lesssim \Phi_{0} / \lambda^{2}$, in the presence of pinning centers. Within the approximation of randomly placed vortices we obtained the relation

$\mathbf{f}_{M}=-\frac{\gamma M \Phi_{0}^{2} \sin ^{2} \theta}{8 \lambda^{4} \omega_{F}^{2}}\left[1+\left(\frac{V_{L}}{\lambda \omega_{F}}\right)^{2}\right]^{-3 / 2} \Theta\left(V_{L}-V_{\mathrm{th}}\right) \mathbf{V}_{L}$

for $\lambda \gg L$. Here $V_{\text {th }}=2 \omega_{F} L$ is the magnon generation threshold velocity. The main consequence of Eq. (9) is that on the $I-V$-curve a step-like increase of the current should be observable at the electric field $E=V_{\mathrm{th}} B_{0} / c$.

Within the last decade significant progress has been made in the fabrication and characterization of ferromagnet/superconductor (FS) superlattices - see Ref. [25] and references therein. Our results can be also applied to such structures provided that the Abrikosov vortices (which are now stacks of pancakes) are oriented strictly perpendicular to the layers and the period of the structure $d$ is much smaller than all characteristic in-plane length scales, which are typically the inter-vortex distance and the domain wall thickness $L$ of the magnetic layers. To obtain the correct expressions for $\mathbf{f}_{M}$ in the FS multilayers two substitutions should be made in Eqs. (6)-(9). First, the force $\mathbf{f}_{M}$ (or the viscosity $\eta_{M}$ ) should be multiplied by $d_{F} / d$, where $d_{F}$ is the thickness of the ferromagnetic layers, due to the partial filling of the structure with magnetic moments. Second, $\lambda$ should be replaced by the London penetration depth of a layered superconductor, $\lambda_{\text {eff }}=\lambda\left(d / d_{S}\right)^{1 / 2}$, where $d_{S}$ is the thickness of the superconducting layers.

We are grateful to L. Bulaevskii for useful discussions and valuable comments. This work was supported in part by the Russian Foundation for Basic Research, European IRSES program SIMTECH (Contract No. 246937), the French ANR program "electroVortex", LabEx "Amadeus" program, and NanoSC COST Action MP1201.

\section{References}

[1] J. Flouquet, A. Buzdin, Phys. World 15 (2002) 41

[2] S.S. Saxena, P. Agarwal, K. Ahilan, F.M. Grosche, R.K.W. Haselwimmer, M.J Steiner, E. Pugh, I.R. Walker, S.R. Julian, P. Monthoux, G.G. Lonzarich, A. Huxley, I. Sheikin, D. Braithwaite, J. Flouquet, Nature 406 (2000) 587.

[3] Dai Aoki, Andrew Huxley, Eric Ressouche, Daniel Braithwaite, Jacques Flouquet, Jean-Pascal Brison, Elsa Lhotel, Carley Paulsen, Nature 413 (2001) 613.

[4] N.T. Huy, A. Gasparini, D.E. de Nijs, Y. Huang, J.C.P. Klaasse, T. Gortenmulder, A. de Visser, A. Hamann, T. Görlach, H.v. Löhneysen, Phys. Rev. Lett. 99 (2007) 067006.

[5] N.T. Huy, D.E. de Nijs, Y.K. Huang, A. de Visser, Phys. Rev. Lett. 100 (2008) 077002.

[6] C. Paulsen, D.J. Hykel, K. Hasselbach, D. Aoki, Phys. Rev. Lett. 109 (2012) 237001.

[7] S. Nandi, W.T. Jin, Y. Xiao, Y. Su, S. Price, D.K. Shukla, J. Strempfer, H.S. Jeevan, P. Gegenwart, Th. Brückel, Phys. Rev. B 89 (2014) 014512.

[8] A.I. Buzdin, JETP Lett. 40 (1985) 956.

[9] V. Braude, E.B. Sonin, Phys. Rev. Lett. 93 (2004) 117001.

[10] V. Braude, Phys. Rev. B 74 (2006) 054515.

[11] N.A. Logoboy, E.B. Sonin, Phys. Rev. B 75 (2007) 153206

[12] V. Braude, E.B. Sonin, Europhys. Lett. 72 (2005) 124

[13] T.K. Ng, C.M. Varma, Phys. Rev. B 58 (1998) 11624

[14] A.A. Bespalov, A.I. Buzdin, Phys. Rev. B 87 (2013) 094509.

[15] L.N. Bulaevskii, M. Hruška, M.P. Maley, Phys. Rev. Lett. 95 (2005) 207002.

16] A. Shekhter, L.N. Bulaevskii, C.D. Batista, Phys. Rev. Lett. 106 (2011) 037001.

[17] Shi-Zeng Lin, Lev N. Bulaevskii, Phys. Rev. B 85 (2012) 134508.

[18] L.N. Bulaevskii, Shi-Zeng Lin, Phys. Rev. B 86 (2012) 224513.

[19] Lev N. Bulaevskii, Shi-Zeng Lin, Phys. Rev. Lett. 109 (2012) 027001.

[20] S.-Z. Lin, L.N. Bulaevskii, C.D. Batista, Phys. Rev. B 86 (2012) 180506 (R).

[21] E.B. Sonin, K.B. Traito, Phys. Rev. B 50 (1994) 13547.

[22] L.P. Gorkov, N.B. Kopnin, Usp. Fiz. Nauk 116 (1975) 413; L.P. Gorkov, N.B. Kopnin, Sov. Phys. Usp. 18 (1975) 496.

[23] L.N. Bulaevskii, A.I. Buzdin, M.L. Kulic, S.V. Panjukov, Adv. Phys. 34 (1985) 176

[24] T.L. Gilbert, IEEE Trans. Magn. 40 (2004) 3443.

[25] M.A. Uribe-Laverde, D.K. Satapathy, I. Marozau, V.K. Malik, S. Das, K. Sen, J. Stahn, A. Rühm, J.-H. Kim, T. Keller, A. Devishvili, B.P. Toperverg, C. Bernhard, Phys. Rev. B 87 (2013) 115105. 